\title{
BMJ Open Percutaneous coronary intervention patients' and cardiologists' experiences of the informed consent process in Northern England: a qualitative study
}

Joy Probyn, ${ }^{1}$ Joanne Greenhalgh, ${ }^{2}$ Janet Holt, ${ }^{3}$ Dwayne Conway, ${ }^{4}$ Felicity Astin ${ }^{5,6}$

To cite: Probyn J, Greenhalgh J, Holt J, et al. Percutaneous coronary intervention patients' and cardiologists' experiences of the informed consent process in Northern England: a qualitative study. BMJ Open 2017;7:e015127. doi:10.1136/ bmjopen-2016-015127

- Prepublication history and additional material is available. To view please visit the journal (http://dx.doi.org/ 10.1136/ bmjopen-2016-015127).

Received 9 November 2016 Revised 8 March 2017 Accepted 24 March 2017

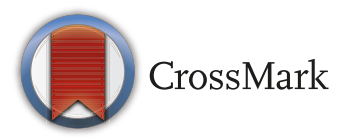

${ }^{1}$ Department of Social Policy, School of Nursing, Midwifery, Social Work and Social Sciences, University of Salford, Salford, UK

${ }^{2}$ School of Sociology and Social Policy, University of Leeds,

Leeds, UK

${ }^{3}$ School of Healthcare, University of Leeds, Leeds, UK

${ }^{4}$ Department of Cardiology, Pinderfields Hospital, Mid Yorkshire Hospitals NHS Trust, Wakefield, UK

${ }^{5}$ Centre for Applied Research in Health, School of Human and Health Sciences, University of Huddersfield, Huddersfield, UK

${ }^{6}$ Research and Development, Calderdale and Huddersfield NHS Foundation Trust, Huddersfield, UK

Correspondence to

Prof. Felicity Astin; f.astin@hud. ac.uk

\section{ABSTRACT}

Objective Informed consent is central to ethical medical practice, but little is known about how the process takes place in clinical practice. Percutaneous coronary intervention $(\mathrm{PCl})$ is a common revascularisation procedure. Studies report that patients overestimate benefits, forget the risks and are unaware of alternative treatments. The aim of this study was to describe PCl patients' and cardiologists' experiences of the informed consent process in acute care settings.

Design/setting/participants A qualitative study with a maximum variation sample of elective and acute $\mathrm{PCl}$ patients and cardiologists taking their consent, recruited from a district general hospital and tertiary centre. Indepth interviews were conducted, and consent discussions were audio recorded. Data collection, coding and theorising occurred simultaneously.

Findings Forty-one (26 male) patients scheduled for elective (20) or urgent (21) PCl and 19 cardiologists (5 female) participated. Despite diversity in patients' experiences of informed consent, elective and acute patients experienced a common four-stage process of consent. Most patients made the decision to have treatment at PCl referral and took a passive role in the discussions we recorded. They recognised cardiologists as experts, trusted the medical system to 'fix' their health problem and were unaware of their role in the informed consent process. Informed consent discussions functioned as a formal 'event', enabling cardiologists to check patients' understanding and enabling patients to access treatment.

Conclusions The configuration of services and patients' perceptions of their role in informed consent underpin a mismatch between legal and ethical principles of informed consent and current practice. The variation in patients' experiences of the current place of informed consent in service delivery represents a missed opportunity for cardiologists to work in decision-making partnerships with patients. In light of recent changes in the law, a new approach to informed consent is required.

\section{INTRODUCTION}

Percutaneous coronary intervention (PCI) (also known as coronary angioplasty) has become one of the most common medical procedures worldwide. ${ }^{1}$ PCI is a
Strengths and limitations of this study

- This study provides a robust, in-depth account of patients' and cardiologists' experiences of the inhospital informed consent process for percutaneous coronary intervention in England.

- Findings can be used to inform service improvements with educational interventions to support the comprehensive implementation of professional and legal frameworks in practice.

- Findings add a new perspective to current knowledge on this topic, which largely reflects an American viewpoint.

- The study focuses on the in-hospital informed consent process, which does not fully account for earlier discussions that may have occurred.

revascularisation technique which is used either electively, to treat angina symptoms in patients with stable coronary artery disease, or urgently, for those with acute coronary syndrome. ${ }^{2}$ A key advantage of PCI is that when successful it improves myocardial perfusion without the need for coronary artery bypass surgery which has a prolonged recovery period. Serious complications are uncommon, but death is one of them. ${ }^{3}$

Patients are required to give informed consent in advance of any medical procedure. Informed consent constitutes good medical practice, ${ }^{4}$ is a universal patient right ${ }^{5}$ and reflects key ethical principles. ${ }^{6}$ The partnership and collaboration between the doctor and patient in supported decision-making is central to this process. For consent to be valid it must be freely given by patients who are informed and deemed to have capacity. In being fully informed patients should understand the full range of treatment options including the risks, benefits, alternative treatments and the outcome should they decide to have no treatment. Although it should be presumed that a patient wishes to be fully 
informed about the risks and benefits, patients' specific information preferences should be respected and documented. ${ }^{7}$ While legal frameworks differ across countries, the recent judgement in Montgomery $\mathrm{v}$ Lanarkshire Health Board $^{8}$ will have a significant impact on discussions about risk that are both fact and case specific during the informed consent process. Consent conversations will now need to demonstrate discussion about material risk, that is any risk that a 'reasonable' person, in the patient's position, would consider important in making their decision about their treatment.

Studies that describe PCI patients' perceptions about their treatment show that they tend to overestimate the benefits, ${ }^{9-11}$ forget the risks and are often unaware of alternative treatments, ${ }^{9}$ suggesting that treatment decisions may not be fully informed or that recall is poor. Theoretical models of the informed consent process acknowledge that informed consent can be perceived as a 'single event' or an ongoing 'process', with the latter perceived as being optimal. ${ }^{12}$ The 'single event' model is unlikely to foster a doctor-patient partnership that enables supported decision-making ${ }^{13}$ and such discussions often occur once the patient has already committed to surgery. ${ }^{12-14}$ Informed consent practice varies widely across hospitals and treatments, ${ }^{11}{ }^{15}$ and the amount and quality of information provided to patients is inconsistent. ${ }^{16}$ The process is complex and often involves interactions with several health professionals. The features of such discussions, including how risk is explained, influence patients' decision-making. ${ }^{17} 18$ The quality of the PCI consent process is influenced by individual and contextual factors such as a lack of time, patients' unwillingness to participate in the process and the expertise of participating doctors. ${ }^{19}$

In summary, we know that the PCI informed consent process is complex and variable and could be improved. To identify how we can optimise informed consent for PCI we need to understand the experiences of those involved, in the context in which the interactions take place. Given this gap in knowledge, the aim of this study was to describe PCI patients' and cardiologists' experiences of the informed consent process in acute care settings. This is the first qualitative study that describes patients' and cardiologists' experiences of the PCI informed consent process.

\section{METHODS}

\section{Study design}

A qualitative methodology was appropriate to explore how PCI informed consent took place. ${ }^{20}$ Principles of constructivist grounded theory were used to generate an understanding of the phenomenon. ${ }^{21}$ In-depth interviews were chosen as the most appropriate qualitative methods and these data were triangulated with consent discussions that occurred when the consent form was signed.

\section{Sample, setting and recruitment}

Following ethical approval we planned a series of in-depth interviews with a maximum variation sample of patients and cardiologists, recruited from two centres. We purposefully selected sites that differed in their service provision to provide a diversity of participant experiences; a district general hospital $(<400$ PCI procedures annually) and a tertiary PCI centre $(<1900$ PCI procedures annually). Participant recruitment was conducted sequentially across sites and continued until no new themes were evident in the interviews. All cardiologists at both sites were invited to participate by a researcher not involved in direct care provision. All eligible patient participants $(<18$ years of age receiving PCI electively or urgently for non-ST elevation myocardial infarction) were either sent study information in advance, or approached by a research nurse prior to giving informed consent. Patient participants were recruited purposively to ensure maximum variation with regard to characteristics which may have potentially influenced their experiences: age, gender and elective or acute treatment.

\section{Data collection}

All participating cardiologists audio recorded the informed consent discussion during which the consent form was signed, with participating patients. We then subsequently interviewed these patients and their cardiologists, using the consent discussion as a starting point for the interview. Participants completed a brief questionnaire (age, gender, procedure, occupation). For consistency one experienced postdoctoral researcher (JP), with training in qualitative research methods, who was not involved in care provision, conducted all semistructured interviews between April 2014 and March 2015. Patients and cardiologists were interviewed in a setting of their choice (hospital or home-patients only) with patient participants interviewed up to 14 days post-PCI. Semistructured interview guides were developed from existing literature and piloted with service users and cardiologists (see online supplementary material 1). Patient interviews explored not only the experiences of the consent discussion that was audio recorded but also their views of the wider consent process. Cardiologists recorded informed consent discussions in the clinical setting; consent forms were signed at this time. All interviews were audio recorded and professionally transcribed verbatim. Field notes and memos were recorded after each interview, including initial observations and reflections about emerging codes and concepts. These data informed the initial coding. Data analysis and participant recruitment took place simultaneously, to allow for the exploration of emerging concepts in keeping with constant comparison methodology. ${ }^{22}$ A group of five cardiology service users provided feedback on the findings.

\section{Data analysis}

Data analysis was conducted by JP, FA and JG. During this process transcripts were independently read and 


\begin{tabular}{ll}
\hline Table $1 \quad$ Participant characteristics & \multicolumn{1}{l}{ n (\%) } \\
\hline Characteristics & \\
\hline Elective patients (SCAD) & $20(49)$ \\
\hline Participants & $12(60)$ \\
\hline Male (\% elective patients) & $64 / 36-79$ \\
\hline Age (mean/range) & \\
Acute patients (NSTEMI) & $21(51)$ \\
\hline Participants & $14(67)$ \\
\hline Male (\% acute patients) & $10(47)$ \\
\hline 'Treat and send' patients & $69 / 40-87$ \\
\hline Age (mean/range) & \\
\hline Cardiologists & $19(100)$ \\
\hline Participants & $13(69)$ \\
\hline Operating interventional cardiologists & $14(75)$ \\
\hline Male & $45 / 30-57$ \\
\hline Age (mean/range) &
\end{tabular}

NSTEMI, non-ST elevation myocardial infarction; SCAD stable coronary artery disease.

'Treat and send' patients were admitted to a non- $\mathrm{PCl}$ centre hospital before being transferred to one of the participating hospitals for treatment.

reread and all transcripts open coded by JP. The analytical process was progressed through a series of meetings with researchers, health professionals and service users. A coding framework was developed which was then systematically applied to the corpus of data. Contributors discussed their own personal views at the meetings and how these might influence the analytical process, to support reflexivity. A process of constant comparison characterised the analysis ${ }^{22}$ supported by qualitative software (NVivo V.10) to manage and retrieve data. This process allowed comparison of the informed consent process, behaviour and perceptions of the participants including deviant case analysis. The coding process was supported by written memos, which underpinned the development of higher level codes, themes, categories and the resulting core category. The methodology was reported using Consolidated Criteria for Reporting Qualitative Research (COREQ) criteria (see online supplementary material 2). ${ }^{23}$

\section{FINDINGS}

Ninety-eight eligible patients and 22 cardiologists were invited to participate. Patient and cardiologist interviews lasted an average of 37 minutes and 42 minutes respectively. The pre-PCI consent discussion between patients and cardiologists lasted an average of 7 minutes. The final sample (see table 1) comprised 41 patients $(42 \%$ response rate) and 19 cardiologists (13 consultant cardiologists and 6 cardiology registrars; $86 \%$ response rate). Informed consent discussions were audio recorded for 37 of the 41 patient participants. Thirteen acute patients withdrew before interview, nine changed their mind and four were not contactable.

We identified three themes and six categories describing the role of the informed consent discussion in patients' decisions to have PCI treatment (table 2). These themes underpinned an overarching category, "I just want to be fixed". In summary, the informed consent discussion that took place in hospital did not facilitate partnership and supported decision-making. Instead it represented a confirmation exercise enabling doctors to encourage continued compliance with treatment, and for patients to access to treatment.

\section{THEME 1: THE INFORMED CONSENT EXPERIENCE}

This theme describes how the informed consent process takes place. During the discussions, cardiologists delivered and patients received information about risks and benefits and checks were made to establish whether patients were satisfied with this information. Patients' experiences of the informed consent process varied according to their clinical presentation (elective or acute) and the hospital they attended, but the decision to have treatment, had in most cases, already been made before the discussion took place.

\section{Category 1: diverse consent journeys}

The pattern of service delivery varied by both procedure and site but all patients, regardless of acute or elective presentation described experiencing the four stages of consent shown in figure 1. There were three 'types' of patient journey: acute (admitted to hospital urgently with myocardial infarction and retained on ward for PCI treatment 12-48 hours later) (female acute patient 6, table 2); 'treat and send' (admitted to a non-PCI hospital urgently with myocardial infarction and retained on ward until transfer to Hospital 1 or 2 for PCI treatment 1-7 days later) (male 'treat and send' patient 33, table 2; and elective (referred to cardiologist by a general practitioner (GP) with angina symptoms and referred for elective PCI) (interventional cardiologist 3, table 2)

Patients accessing acute PCI treatment had their first contact (stage 1: diagnosis, fig 1) with a health professional either by ambulance, through accident and emergency, or were transferred from another hospital in the region. Patients had a preliminary consultation in a ward setting with a general cardiologist or in a minority of elective cases at one hospital, the interventional cardiologist, that would conduct the procedure (stage 2: PCI referral, fig 1) and it was at this consultation that the decision to have treatment was made. They were given written information about the procedure to read and this was followed by 'detailed information giving (stage 3: detailed information giving, fig 1)' discussion 2-24hours later. The recorded discussion took place at this point at hospital 1. Practices varied regarding the time at which the consent form was signed. This could occur either during the in-depth discussion, or when 
Table 2 Themes and categories

"I just want to be fixed"

Theme 1: the informed consent experience

Diverse consent "When I came to the hospital they told me that I probably had a few small heart attacks. I was given journeys information. But to tell you the truth I may have glanced at it. I don't really know whether I went into it with a fine toothcomb but I was aware of it anyway... Before the angioplasty the doctor went through the consent form. But the thing is the amount of information that's fed to you is enough to frighten you to death actually. I think maybe it's a little bit too much" (female acute patient 6, interview).

"Seven days I was waiting at (admittance hospital) before they took me over to (treating hospital) for surgery. It was ridiculous, what a waste of money keeping me in, but if they'd have discharged me I would have been back of the list. I had some information about angioplasty at (admittance hospital) but signed the consent form at (treating hospital) and saw the doctor there just before" (male 'treat and send' patient 33, interview).

"With the elective patients, about six or seven people will have spoken to them about the procedure before you stick a needle in them. So they get enough time... slightly less discussion with acute coronary syndrome patients because they are in the hospital rather than coming in to clinics as an outpatient" (interventional cardiologist 3, interview discussing elective patient journey).

Information transfer

"The most common risks are bruising and bleeding, either from the wrist or from the groin. And usually we can just treat that with pressure. Occasionally, we damage the artery and that might need a surgical repair. Other problems are, you can be allergic to the dye but we know you had it last time so I don't think you'll be allergic to it. The dye can cause problems with your kidneys and you have diabetes so you need to make sure you're well hydrated afterwards... "(interventional cardiologist 11, audio recorded consent discussion).

"I'm fairly happy and confident in consenting almost all patients who come in for angioplasty, I think I've assisted and seen quite a few cases, but there are still cases where I might not be able to quote an accurate risk, especially in someone who's undergoing a very risky procedure" (cardiology registrar 2 , interview).

JP: "Do you think that patients want to know about the risks?"

"Some people will just outright say, 'I don't want to know about the risks'. I accept that and say 'that's fine'... it's wrong to say you might die, you might have a heart attack, you might have a stroke. They just don't want to know. They will say, 'right I trust you; you get on and do what you think you need to do'. I think that is perfectly reasonable" (interventional cardiologist 6, interview).

"So there are three ways we can treat that. Tablets, heart bypass surgery or angioplasty. It's not that easy to justify a major operation which carries quite a risk and obviously the older you get that risk is just a little bit more. So the other two options are either tablets or stents, the angioplasty. Okay. That's unblocking it with the balloons and the stents. I think we could be fair to say the tablets haven't really worked. From the investigation you had last week we know what needs to be done (angioplasty)"(interventional cardiologist 4 , audio recorded consent discussion).

"The only thing was at pre-op assessment I was told about heart attack, a stroke and mortality all in the same sentence. I wanted to know what the separate risks for those things... but they didn't have that information... when I got to see doctor $X$ (on the day of the procedure), he got the information straight away but that was right before I went in" (male elective patient 9, interview).

\section{Theme 2: role and expectations}

'A formal event' "We often only interact with the patient on the day of the procedure... by the time they come to the catheter lab...somebody should have gone through what we're doing and why we're doing it" (interventional cardiologist 14, interview).

"Patients usually want to make sure that I'm going to do the procedure or not. In most of the cases I'm consenting (completing the consent form) and someone else is doing the procedure so after our conversation they will see the consultant who is going to do the procedure and the patient will feel more confident about the doctor and they can confirm everything for them" (general cardiologist 7, interview). "The surgeon came and gave me booklets and wanted me to read them. I said to him, 'Look, do you know what you are doing? If you do, just get on with it. I do not want any of this nonsense.' If there was a form to sign I signed it, and that was the end of it" (male elective patient 5, interview).

"He probably thinks, "I've lived a lot of my life and I've got to this point now without worrying about red tape". They often see it as red tape and paperwork. I think he was probably thinking that this was all political correctness and red tape but he was thinking "I've got to where I am without bothering about this; let's just get on with it" (interventional cardiologist 1, interview). 
Table 2 Continued

"I just want to be fixed"

Expectations of treatment
"I decided to have it as soon as doctor $X$ said about it because there was so much pain for me attached to the simple things I was doing, walking downhill and so the choice never entered my mind. I was just quite happy to go along with what they recommended, you know I just wanted my old life back" (female elective patient 21, interview).

"It was a one way street for me and it was going to stop me having another heart attack. I want to stay alive and that's it really" (male acute patient 11, interview).

"I knew what it was all about ...he explained it all to me, and I said yes, and I just signed the consent form, because I had nothing to worry about if I knew doctor $X$ were there, because I mean he had done it before" (male acute patient 15, interview).

"I fully recognised what they were doing and when they didn't (put a stent in) I was upset because I thought they would do it. But we are back to square one and so I have six weeks of these tablets and take it from there... but I don't want a bypass at 80 odd years old" (male elective patient 8, interview).

\section{Theme 3: deciding to have treatment}

'You're the expert Doctor: Is there anything else you want to ask?

doctor

Patient: "It's really about what I'm going to feel like after it's been done. I need to get back to work, I'm a policeman...I've been off since the end of September...so how quickly am I going to be able to get back to work, really?

Doctor: "It depends what...how much we do today. So if we get all the arteries open today, then in terms of physical activity, l'd be pretty happy for you to return to physical activity almost immediately...." (male elective patient 36 and interventional cardiologist 13, audio recorded consent discussion).

"I'm a firm believer in if the medical professional can give me a decent reason, or a reasoned explanation for doing something, then I'm quite happy to go along with it. I'll do a little bit of research...but I'm prepared to be guided by what the doctors say. What's the point in saying no? You've got to gulp and get on with it, they know best" (female elective patient 18, interview).

"There was no decision for me to make. The doctors recommended that's what needed to be done. And there's no point it crossed my mind not to have it, no. Never. The risks do cross your mind but they don't take more than two seconds to weigh up the pros and cons and come out with an answer"(female elective patient 22, interview).

A 'Hobson's 'So if you know there's risk, you decide either yes to have it done or no. And the thing is you've got to have choice'

"I knew how I was feeling and I couldn't cope very well with the feelings I was having, getting out of breath and things like that. So I thought well I haven't really got an option" (female elective patient 24, interview). Well, the decision was made beforehand because doctor $X$ said to me, when I went to see him, he says, "We're going to have a look inside your heart." He says, 'We'll put a camera in and this, that and the other'and I just turned round to him, I says, 'Well, whatever's got to be done, do it. 'You know, there's no choice really. l'd sooner you do it than mess about for four or five months and then come back later. Do it. You know, do it straight away, don't hang about. Just do it, and that's what they've done" (male elective patient 10, interview).

consent was confirmed, just before treatment. Patients transferred from non-PCI hospitals ('treat and send' patients) waited between 1 to 7 days at the admitting hospital before a bed was available at the hospital with PCI services. The duration of the wait depended on the throughput of the hospital providing PCI services. The operating cardiologist recapped information immediately before the procedure and confirmed the patient wanted to proceed (stage 4: confirming consent, fig 1). The recorded discussion took place here at hospital 2.

Elective patients had a similar 'journey' except the process took place over a longer period and the settings were different. First contact was typically through a GP (stage 1: diagnosis, fig 1). Preliminary consultation took place in an outpatient setting and it was usually at this consultation that the decision to have treatment was made (stage 2: PCI referral, fig 1). Following this they were sent written information about PCI by post.
Attendance at a pre-PCI clinic facilitated 'detailed information giving' (stage 3: detailed information giving, fig 1) which could be nurse-led, or doctor-led, with the latter including the completion and signing of the consent form. The recorded discussion took place at this point at hospital 1. As with acute PCI patients, the final part of the informed consent process was 'confirming consent' (stage 4: confirming consent, fig 1) conducted by the operating cardiologists just before treatment. The recorded discussion took place at this point at hospital 2. The consent form was signed at different stages at the two sites, either at the preop assessment, or immediately before the procedure. The patient experience from the first point of contact to the PCI procedure was thus influenced by a number of factors, including the amount of time between referral and treatment, the health professionals they had faceto-face contact with, whether they were admitted to 




Figure 1 The four-stage informed consent process experienced by acute and elective patients at both hospital sites. Key: A\&E, accident and emergency; GP, general practitioner; $\mathrm{PCl}$, percutaneous coronary intervention.

a PCI treatment hospital in the first instance and the point at which the consent form was signed.

\section{Category 2: information transfer}

The breadth of risk information given to patients in the discussions varied considerably based on cardiologists' judgements about what level of information was appropriate or comfortable for them to disclose, the amount of time available for the discussion and their knowledge of the patient. Most cardiologists balanced the risks of the procedure with a statement about the benefits (Interventional cardiologist 11 , table 2). Some gave patients a choice as to whether they wanted the information about risk whereas others felt duty-bound to disclose it regardless. In one case a patient requested not to be given any information about risk and the cardiologist respected this choice.

Risk was rarely individualised for patients even when they had multiple health conditions or had had previous coronary bypass surgery. There was variation in the degree of detailed information about risk given to patients (see table 3). At hospital 1, the consent forms were pre-printed with general risk information. This information was provided as it was usual practice for general and registrar cardiologists to take consent rather than interventional cardiologists. At hospital 2 where interventional cardiologists took consent, cardiologists documented risk calculations on the consent form by hand.
General and registrar cardiologists (those taking consent from patients but not performing the intervention) did not always have the specialist knowledge required to individualise risk information (cardiologist registrar 2 , table 2) and interventional cardiologists were constrained by the short amount of time available for the discussion, particularly as some conducted the discussion immediately before the procedure. Some patients would rather not have known about risks (interventional cardiologist 6 , table 2); others wanted to know every detail. Some patients, who were admitted acutely, struggled to absorb information, probably due to the stress of their emergency admission to hospital. In contrast, some elective patients felt that knowing too much information early on in the process caused them anxiety. Most of the patients who did not want to know about the risks also did not question the doctor's decision to refer them for treatment. Those who did wish to know about the risks were more proactive in their decision-making and recognised their role in the process. Regardless of the mode of delivery and content of the information given, it was clear that the value patients placed on the benefits of treatment clearly outweighed the risks.

Alternative options for treatment were not usually discussed in stage 4 'confirming consent' (fig 1) of the informed consent discussions with elective or acute patients. When options were mentioned, they were acknowledged as potential options to eliminate rather 
Table 3 Variations in descriptions of risk by two different cardiologists (acute cases). P (patient); C (cardiologist)

\section{Detailed risk description}

(female acute patient 19 (P); cardiologist 7 (C):hospital 1)
Less detailed risk description (female acute patient 24 (P); cardiologist 15 (C):hospital 2)

\begin{abstract}
C: "On average the risks of stretching an artery and putting a stent is about $1 \%$ or $2 \%$ risk. So one or two people in a 100 of us causing a problem, of causing a heart attack. On average the sorts of narrowing that we would treat would have a high risk of causing a heart attack if we didn't treat. So there's some risk for the procedure but there is as much or more risk without the procedure. There are other risks that I mentioned before things like strokes, allergic reactions, kidney problems are still very rare. So there's still less than a $1 \%$ likelihood. So overall your risk of any major problems, stroke, heart attack, life threatening problem and dying from the procedure is very unlikely. The risk of us causing a bit of injury to a heart though and triggering a heart attack is maybe about $1 \%$ or $2 \%$. It's not usually a life changing event but it can happen during the procedure. We quote with an angioplasty about a 1 in 1000 risk of needing a bypass as an emergency. That's still the case for you even though you've had a bypass because occasionally the catheter might injure the artery and you might need something done quite quickly, which might need surgery rather than stents. But it is very, very rare and it is 1 in 1000 less that needs that.

$P$ : "Okay, yes."

C: "As in any procedure, there is always a risk.

$P$ : "Just ...just don't burst my artery that is all [laughs].

C: "You do know...yes, certainly, you do know one of the risks but there are several risks but the risks are very small. $P$ : "Yes.

C: "So the risks are one, as you said, you can have bleeding from either the site, from the groin or from the arm; there is a small risk of you having a stroke or a heart attack during the procedure, it is very tiny.

$P$ : "Yes.

C: "There is a tiny risk that the contrast that we inject can cause impairment of your kidney but that usually recovers in a few days with fluids. Very few people are allergic to contrasts, not many, but of course the benefit of this thing is tremendous, if you do have a blockage. The benefit of doing this procedure is

tremendous, that is why we go ahead and do it."
\end{abstract}


5 and interventional cardiologist 1, table 2) and to protect the doctor against litigation. Patients were anxious about their health, focused on getting well and felt obliged to accept the treatment offered. A small minority of patients defined it as a decision-making exercise; in one case this was because the patient was given a choice between bypass surgery and PCI. Although this patient was given adequate information, he was not confident he had made the 'right' decision, indicating the importance of the doctor-patient partnership in decision-making.

\section{Category 2: expectations of treatment}

Elective and acute patients had different expectations of treatment based on the information they had been given, although both types of patients focused on the outcome of treatment, rather than the treatment itself. Elective patients focused on getting 'back to normal' and improving their quality of life with reduced angina symptoms and increased energy (female elective patient 21, table 2), whereas acute patients focused on staying alive and prevention of future heart attacks (male acute patient 11, table 2). A small number of patients who had undergone previous angiograms, felt reassured and knew what to expect (male acute patient 15, table 2). Patients were disappointed when their expectations of treatment were not achieved, and they were not 'fixed'; for example in two cases where PCI could not be done and other options such as a bypass were being explored (male elective patient 8 , table 2 ).

\section{THEME 3: DECIDING TO HAVE TREATIMENT}

This theme explains how patients' perceptions of doctors and the availability of medical treatment influenced their decisions to have PCI treatment. Patients perceived medical authority to override their autonomy for decision-making and felt obliged to accept National Health Service (NHS) treatment recommended to them if they had the will to get well. This meant that the informed consent discussion was often an information delivery exercise rather than a supported decision-making discussion, as the decision had usually been made at the referral stage (stage 2 , fig 1 ).

\section{Category 1: 'You're the expert doctor'}

Cardiologists took a leading role in discussions that occurred during the signing of the consent form; most were short, concise and standardised, with little involvement from the patient. Some lasted as little as 3 minutes when occurring immediately prior to the procedure. It was unusual for patients to ask questions even when prompted by the doctor. Patients who asked questions were motivated by contextual factors such as the impact of the procedure on caring responsibilities and their career (male patient 36 and interventional cardiologist 13, table 2). Patients distinguished levels of expertise between themselves and cardiologists. Most patients felt they did not need information about the procedure on the basis that they trusted the doctor, and that it was their role to listen but not to question (female elective patients 18 and 22, table 2). Patients and cardiologists valued the interaction during the discussion for different reasons. For interventional cardiologists it was important to meet their patients to develop a 'rapport' with them before the invasive procedure. For patients, having the procedure explained to them by a doctor was the authority confirming that the procedure would be carried out.

\section{Category 2: a 'Hobson's choice'}

Patients talked about having a 'Hobson's choice' (a phrase describing the choice between accepting or refusing only one available option) to make about treatment. This was especially prevalent when the patient was told that a bypass and/or medication alteration were not options for them early on in the informed consent process, or when acute patients were told that it was necessary to prevent future risks of a heart attack (patients 20, 24 and 10, table 2). They acknowledged they were given adequate information to make a decision, but ultimately they felt that if they wanted to be well again, they had an obligation to accept the treatment recommended to them. Thus, most patients made the decision to have treatment at stage 2 (referral, fig 1). Interestingly, elective and acute patients both felt this, although the outcome of the elective procedure was solely symptom improvement rather than the prevention of future heart attacks. This indicates that when the in-depth discussion and consent form signing occurs at stages 3 or 4 (fig 1), the decision to have treatment has already been made. Some cardiologists believed that elective patients were more likely to have made the decision to have treatment before the discussion than acute patients, given the longer duration of time they had to consider.

\section{CORE CATEGORY: 'I JUST WANT TO BE FIXED'}

The informed consent discussion was part of a four-stage process of informed consent taking place either at the patient's preop assessment (elective), or immediately before the procedure (acute). Patients' journeys prior to the consent discussion involved discussions with a range of different health professionals but varied based on symptom presentation and the hospital attended. In most cases the discussions we recorded did not support decision-making as the decision had already been made at stage 2 (PCI referral, fig 1). Rather, discussions acted as a formal event to check patient understanding for cardiologists and as access to treatment for patients. Patients wanted to be 'fixed', trusted the medical profession and felt obliged to accept the treatment offered. Cardiologists (particularly those taking consent immediately before the procedure) had rarely met patients prior to the discussion. This meant they had limited time with, and knowledge of, patients and had to make assumptions about the information patients had been given prior to the discussion. This was particularly difficult when dealing with 'treat and send' patients from another hospital as cardiologists 
were unaware of earlier interactions and discussions they may have had.

In general, patients were passive in decision-making during the consent discussions we recorded as they believed they had no other choice, trusted medical staff to 'fix' them and ultimately had already made the decision to have treatment. This underpins the overall theory that the discussions we recorded (when the consent form was signed) functioned as a pre-treatment gate-keeping exercise for both parties. Such discussions enabled the 'fixing' of the patient but decision-making takes place at the earlier 'referral' stage.

\section{DISCUSSION}

\section{Summary}

Our findings show that patients had a variety of encounters with health professionals before meeting the operating interventional cardiologist immediately before the procedure. Most had already made the decision to have treatment by this point after reading written information given to them on the ward (acute), or sent to them by post (elective), and having had a referral discussion with a cardiologist. Patients recognised cardiologists as experts and trusted the medical system to 'fix' their health problem. Most were typically unaware of their role in the consent process and had limited autonomy and participation in the consent discussion that occured when the consent form was signed. This informed consent discussion functioned as a formal 'event' enabling cardiologists to check patients' understanding and enabling patients to access treatment.

\section{STRENGTHS AND LIMITATIONS}

This study builds on the limited qualitative research available on informed consent for $\mathrm{PCI}^{12-1419}$ and adds a new perspective to current knowledge on this topic as earlier studies have largely reflected an American viewpoint. ${ }^{91012-141718}$ Our study is original because of the triangulation of data from 'real' informed consent discussions together with in-depth interviews with participants. Our model of the informed consent process adds to, and extends on, existing yet dated research, ${ }^{12-14}$ and is grounded in the reality of the English practice setting. The qualitative nature of the findings may limit transferability to other sites but the triangulation of methods supports the trustworthiness of the study findings. Informed consent is a universal patient right and a process that occurs globally for all invasive procedures. Accordingly it is likely that findings will have application across other healthcare settings in which informed consent is required. We only directly observed one part of patients' informed consent journeys (the signing of the consent form) and we relied on patient and cardiologist reports of earlier discussions and the overview of the consent process. Furthermore, although this was unknown at the time of data collection, the consent discussions were recorded at different points in the consent journey at the two sites. Thus, further research is needed to explore all stages of informed consent to determine whether the current service delivery model supports patients to make fully informed decisions about PCI treatment. Although cardiologists were asked to conduct the informed consent discussions as per standard practice, the audio recording of consultations could affect the nature of the consultation thereby introducing potential bias.

\section{COMPARISON WITH EXISTING LITERATURE}

Our model of the in-hospital informed consent process, based on patients' and cardiologists' accounts, supports an earlier published model. ${ }^{12}$ However, our findings show that the first four stages of Lidz and colleagues' (establishing, responsibility, defining the problem, setting goals for treatment and selecting an approach to treatment) take place in the PCI referral consultation, and patients made the decision to have treatment at this point. Given that previous research has shown that how cardiologists present information has a strong influence on patients' perceptions of PCI treatment, ${ }^{17} 18$ exploring what happens in these initial discussions as part of the informed consent process is vital. In the discussions we recorded, alternative options for treatment were rarely mentioned. Although they may have been mentioned in previous discussions patients had with other health professionals, patients were not always reminded of this before signing the consent form. Other evidence suggests that PCI patients are not always aware of alternative treatments ${ }^{9}$ and that cardiologists' perceptions of the benefits of PCI are more optimistic than the evidence available, with PCI being the preferred treatment despite prognosis. ${ }^{24}$

Risk information given to patients was variable across discussions as shown elsewhere ${ }^{1516}$ but because of its placement in this discussion, it did not influence patients' decisions to have treatment. To confirm understanding before consent, cardiologists asked patients if they understood the information given and usually gave them the opportunity to ask questions. Patients rarely asked questions and this passive role in decision-making could explain why patients in studies of informed consent for various surgical procedures often have difficulty recalling information. ${ }^{25}{ }^{26}$ Passivity in decision-making (ie, feeling they had 'no choice') was underpinned by threatening symptoms, angina pain (elective patients) or a (NSTEMI) heart attack (acute patients) and a desire to get well. Patients had faith in the medical profession to 'fix' them, and saw PCI treatment as their only option. Other studies have reported that patients see PCI as a 'fix ${ }^{, 27}$ and are somewhat reluctant to participate in decision-making discussions. Moreover informed consent discussions studied in other settings, such as non-emergency surgical procedures have been reported to function as an interaction that confirms rather than facilitates supported decision-making. . ${ }^{19}$ When patients see only one realistic option in a health decision, the collaboration envisioned in the literature on supported decision-making is probably unrealistic. ${ }^{28}$ 


\section{IMPLICATIONS FOR RESEARCH AND PRACTICE}

Our findings, and others ${ }^{19}$ illustrate that service delivery and time pressures are key barriers to shared decision-making in informed consent discussions. It is concerning that issues pertinent 20 years ago in Lidz and colleagues' research, ${ }^{12-14}$ could continue to jeopardise the quality of informed consent. Detailed information provided to patients often comes after the decision to proceed with treatment has been made and the patient has committed to the treatment thereby reflecting an 'event' rather than a 'process' model of consent. While this satisfies legal requirements, it does not foster supported decision-making. ${ }^{12-14}$ We have identified a four-stage consent process underpinning patients' otherwise diverse informed consent experiences. We have shown that patients sometimes make decisions to have treatment before having an in-depth discussion and that risk information varies in presentation across sites. Further research is needed to understand the context in which these decisions are made at the PCI referral stage, to ensure that opportunities for supported decision-making are optimised. Educational resources for patients and health professionals are needed to raise awareness of their roles in supported decision-making. Research has shown that decision-making processes are improved when practitioners are motivated and perceive that it will have an impact on the clinical process and patient outcomes. ${ }^{29}$ This is vital as the roles and relationships between doctor and patient can shape the behaviours and attitudes of patients, and support or discourage them to engage in their healthcare. ${ }^{30}$

\section{CONCLUSIONS}

Research has shown that the process of informed consent is fraught with limitations regardless of country and setting. Informed consent is defined as a process to enable patients to make informed decisions about their treatment, yet our findings suggest that in the context of PCI the process acts as a formal event to check understanding and enable access to treatment rather than to support decision-making. Patients' decisions to have treatment are largely based on trust and obligation. Our process model of informed consent builds on, and extends, existing research and indicates that cardiologists may unintentionally support patients to be passive, rather than active, in the decision-making process. The configuration of services and patients' perceptions of their role in informed consent underpin a mismatch between legal and ethical principles of informed consent and current practice. In light of the Montgomery case, ${ }^{8}$ the emphasis of informed consent has shifted away from doctors' judgments about what to disclose, to patients' judgments about what they want to know. In a time of economic restraint in the NHS we need to find ways, through education, to ensure that patients are supported to make decisions about their care. A new approach to informed consent is required. Educational interventions to support decision making have the potential to influence practice internationally. We have focused on just one stage of the informed consent process for PCI. Further work is needed to explore the link between supported decision-making and informed consent through all stages of the consent process to identify strategies for service improvement.

Correction notice This paper has been amended since it was published Online First. Owing to a scripting error, some of the publisher names in the references were replaced with 'BMJ Publishing Group'. This only affected the full text version, not the PDF. We have since corrected theseerrors and the correct publishers have been inserted into the references.

Acknowledgements The authors thank Mr Keith Marshall, Mr David Openshaw and the team of service users for their invaluable input;Mrs Judith Wright and Mr John Humphreys for research support and Dr Alison Morton who contributed to the development of the funding application.

Contributor JP collected the data, led data analysis, drafted the paper, finalised this version and is accountable for all aspects of the work. JG and JH contributed to the design of the work and data analysis, provided critical comments on drafts of the paper, approved the final version and is accountable for all aspects of the work. DC contributed to the design of the work, provided critical comments on drafts of the paper, approved the final version and are accountable for all aspects of the work. FA conceived the idea and led the design and funding application, contributed to data analysis and to writing the paper, approved the final version and is accountable for all aspects of the work.

Funding This paper presents independent research funded by the National Institute for Health Research (NIHR) under its Research for Patient Benefit (RfPB) Programme (Grant Reference Number PB-PG-0712-28089).

Disclaimer The views expressed are those of the authors and not necessarily those of the NHS, the NIHR or the Department of Health. This report contains anonymised excerpts from interviews and discussions conducted during the research.

Competing interests We have read and understood BMJ policy on declaration of interests and declare that we have no competing interests.

Ethics approval This study was approved by Yorkshire \& the Humber Research Ethics Committee (Ref: 13/YH/0418).

Provenance and peer review Not commissioned; externally peer reviewed.

Data sharing statement Patients did not give consent for data to be shared beyond the research team.

Open Access This is an Open Access article distributed in accordance with the Creative Commons Attribution Non Commercial (CC BY-NC 4.0) license, which permits others to distribute, remix, adapt, build upon this work non-commercially, and license their derivative works on different terms, provided the original work is properly cited and the use is non-commercial. See: http://creativecommons.org/ licenses/by-nc/4.0/

(c) Article author(s) (or their employer(s) unless otherwise stated in the text of the article) 2017. All rights reserved. No commercial use is permitted unless otherwise expressly granted.

\section{REFERENCES}

1. Stefanini GG, Holmes DR. Drug-eluting coronary-artery stents. $N$ Engl J Med 2013;368:254-65.

2. Windecker S, Kolh P, Alfonso F, et al. 2014 ESC/EACTS guidelines on myocardial revascularization: the task force on myocardial revascularization of the european society of cardiology (ESC) and the european association for Cardio-Thoracic surgery (EACTS) Developed with the special contribution of the european association of percutaneous cardiovascular interventions (EAPCI). Eur Heart $J$ 2014;35:2541-619.

3. National Institute for Cardiovascular Outcomes Research and British Cardiovascular Intervention Society 2014. National audit of percutaneous coronary interventions: annual public report. 2014 http://www.ucl.ac.uk/nicor/audits/adultpercutaneous/documents/ 2013_annual_report_pdf (accessed 2 Nov 2016).

4. General Medical Council. Consent: patients and doctors making decisions together 2008. http://www.gmc-uk.org/static/documents/ content/Consent_-_English_1015.pdf (accessed 2 Nov 2016).

5. World Health Organisation. Patient's rights. http://www.who.int/ genomics/public/patientrights/en/ (accessed 2 Nov 2016). 
6. Manson NC, O'Neill O. Rethinking informed consent in bioethics. Cambridge: Cambridge University Press, 2007.

7. Department of Health. Good practice in consent implementation guide: consent to examination or treatment. London: DOH, 2009

8. The Supreme Court. Montgomery v Lanarkshire Health Board. 2015 https://www.supremecourt.uk/decidedcases/docs/UKSC_2013_ 0136 Judgment.pdf (Accessed 2 November 2016).

9. Kureshi F, Jones PG, Buchanan DM, et al. Variation in patients' perceptions of elective percutaneous coronary intervention in stable coronary artery disease: cross sectional study. BMJ 2014;349:95309.

10. Lee JH, Chuu K, Spertus J, et al. Patients overestimate the potential benefits of elective percutaneous coronary intervention. Missouri Med 2013;109:79-84.

11. Chandrasekharan DP, Taggart DP. Informed consent for interventions in stable coronary artery disease: problems, etiologies, and solutions. Eur J Cardiothorac Surg 2011;39:912-7.

12. Lidz CW, Appelbaum PS, Meisel A. Two models of implementing informed consent. Arch Intern Med 1988;148:1385-9.

13. Lidz CW, Meisel A, Osterweis M, et al. Barriers to informed consent. Ann Intern Med 1983;99:539-43.

14. Lidz CW. Research on informed consent: unexpected impacts. Am Sociol 1996;27:90-4.

15. Leclercq WK, Keulers BJ, Scheltinga MR, et al. A review of surgical informed consent: past, present, and future. A quest to help patients make better decisions. World J Surg 2010;34:1406-15.

16. Goff SL, Mazor KM, Ting HH, et al. How cardiologists present the benefits of percutaneous coronary interventions to patients with stable angina: a qualitative analysis. JAMA Intern Med 2014:174:1614-21.

17. Rothberg MB, Scherer L, Kashef MA, Coylewright M, et al. The effect of information presentation on beliefs about the benefits of elective percutaneous coronary intervention. JAMA Intern Med 2014;174:1623-9.

18. Gurm HS, Litaker DG. Framing procedural risks to patients: is $99 \%$ safe the same as a risk of 1 in 100? Acad Med 2000;75:40-2.
19. Wood F, Martin SM, Carson-Stevens A, et al. Doctors' perspectives of informed consent for non-emergency surgical procedures: a qualitative interview study. Health Expect 2016;19:751-61.

20. Mays N, Pope C. Qualitative research in health care; assessing quality in qualitative research. BMJ 2000;320:50-2.

21. Charmaz K. Constructing Grounded Theory: a Practical Guide through qualitative analysis. London: SAGE, 2000.

22. Strauss A, Corbin J. Basics of qualitative research: grounded theory procedures and techniques. $2^{\text {nd }}$ edition. London: SAGE, 1990.

23. Tong A, Sainsbury P, Craig J. Consolidated criteria for reporting qualitative research (COREQ): a 32-item checklist for interviews and focus groups. Int J Qual Health Care 2007:19:349-57.

24. Rothberg MB, Sivalingam SK, Ashraf J, et al. Patients' and cardiologists' perceptions of the benefits of percutaneous coronary intervention for stable coronary disease. Ann Intern Med 2010;153:307-13.

25. Shurnas PS, Coughlin MJ. Recall of the risks of forefoot surgery after informed consent. Foot Ankle Int 2003;24:904-8.

26. Vallance JH, Ahmed M, Dhillon B. Cataract surgery and consent; recall, anxiety, and attitude toward trainee surgeons preoperatively and postoperatively. J Cataract Refract Surg 2004;30:1470-85.

27. Astin F, Closs SJ, McLenachan J, et al. The information needs of patients treated with primary angioplasty for heart attack: an exploratory study. Patient Educ Couns 2008;73:325-32.

28. Whitney SN. A new model of medical decisions: exploring the limits of shared decision making. Med Decis Making 2003;23:275-80.

29. Légaré $F$, Ratté $\mathrm{S}$, Gravel K, et al. Barriers and facilitators to implementing shared decision-making in clinical practice: update of a systematic review of health professionals' perceptions. Patient Educ Couns 2008;73:526-35.

30. D'Amour D, Ferrada-Videla M, San Martin Rodriguez L, et al. The conceptual basis for interprofessional collaboration: core concepts and theoretical frameworks. J Interprof Care 2005;19(Suppl 1)116-31. 